\title{
Sistemas agrícolas y silvopastoriles en el Chaco Semiárido. Impactos sobre la productividad primaria
}

\author{
Pablo Baldassini ${ }^{1, \bigotimes}$ \& José M. Paruelo ${ }^{1,2,3}$
}

${ }^{1}$ IFEVA,DepartamentodeMétodosCuantitativosySistemasdeInformación,FacultaddeAgronomía,UniversidaddeBuenos Aires, CONICET. Buenos Aires, Argentina. ${ }^{2}$ Instituto Nacional de Investigaciones Agropecuarias (INIA). La Estanzuela, Uruguay. ${ }^{3}$ IECA, Facultad de Ciencias, Universidad de la República. Montevideo, Uruguay.

\begin{abstract}
Resumen. La productividad primaria neta (PPN) se define como la tasa a la cual se acumula biomasa por unidad de área y de tiempo, y se la considera una variable integradora del funcionamiento de los ecosistemas. El desmonte y los cambios en el uso del suelo que afectan gran parte de la región del Chaco Semiárido pueden impactar sobre las ganancias totales de C y su estacionalidad. Trabajos previos en la región utilizan índices espectrales de vegetación como subrogado de la PPN. Sin embargo, arriban a resultados dispares y sólo se circunscriben a lo que sucede con la parte aérea (PPNA), sin considerar los cambios que ocurren en la porción subterránea (PPNS), que suele variar entre tipos fisonómicos de vegetación. Sobre la base del modelo de eficiencia en el uso de la radiación, de información espectral derivada de sensores remotos y de relaciones PPNS/PPN de la bibliografía, el objetivo de este trabajo fue cuantificar el efecto de la transformación en el uso y la cobertura del suelo sobre la PPN, y su variación estacional. En particular, se evaluó el cambio de la PPN en sistemas agrícolas y silvopastoriles con un componente leñoso variable, respecto del bosque original. En general, se observó que los usos del suelo que presentaban una mayor productividad aérea mostraban, al mismo tiempo, los menores niveles de productividad subterránea. El doble cultivo trigo-maíz presentó la mayor PPN total, significativamente superior a la de los sistemas silvopastoriles entre 5 y $25 \%$ de cobertura arbórea, y a los cultivos de trigo-soja y soja de primera. Sin embargo, su productividad no varió significativamente de la del bosque, las pasturas megatérmicas y el maíz de primera. Los resultados obtenidos no sólo aportan información sustancial para concluir cuantitativamente en términos de la magnitud del cambio en unidades de materia seca, sino que también permiten establecer un orden o ranking más apropiado de las coberturas y usos del suelo en cuanto a la productividad total.
\end{abstract}

[Palabras clave: índices espectrales, modelo de Monteith, eficiencia en el uso de la radiación, sistemas silvopastoriles]

\begin{abstract}
Agricultural and silvopastoral systems in the semi-arid Chaco. Impacts on primary productivity. Net primary production (NPP) is defined as the rate at which biomass accumulates per unit area and time and is considered an integrating variable of the ecosystem function. Deforestation and land use changes observed in the semi-arid Chaco region may affect total $C$ gains and seasonality. Previous work in the region uses spectral vegetation indexes as a subrogate of the NPP. However, they arrive at contrary results and only concluded about to what happens with the aboveground production, without considering the changes that occur in the belowground production, which usually varies between physiognomic types of vegetation. Based on the model of radiation use efficiency, spectral information obtained from remote sensing and BNPP/NPP relationships of the bibliography, the goal of this work was to quantify the effect of the land use and cover change on the NPP and its seasonal variation. Specially, we evaluated the change on NPP in agricultural and silvopastoral systems with a variable woody component respect to the original forest. NPP and its seasonal variation of the main land uses of the semi-arid Chaco region. We observed that, in general, the land uses that showed higher aboveground productivity showed, at the same time, the lower levels of belowground productivity. The double wheat-maize crop presented the highest total NPP, significantly higher than that of the silvopastoral systems between 5 and $25 \%$ of tree cover, and to the wheat-soybean and soybean crops. However, their productivity did not vary significantly from that of the native forest, the tropical pastures and the maize. The results obtained not only provide substantial information to conclude quantitatively in terms of the magnitude of the change in units of dry matter, but also allow to establish a more appropriate order or ranking of land uses in terms of total productivity.
\end{abstract}

[Keywords: spectral indexes, Monteith model, radiation use efficiency, silvopastoral systems]

Editora asociada: María Vanessa Lencinas 凶pbaldass@agro.uba.ar
Recibido: 14 de Mayo de 2019

Aceptado: 6 de Noviembre de 2019 


\section{INTRODUCCIÓN}

La productividad primaria neta (PPN) se define como la tasa a la cual se acumula biomasa por unidad de área y de tiempo (Oesterheld et al. 2010), y es considerada una variable integradora del funcionamiento de los ecosistemas (McNaughton et al. 1989). Fisher et al. (2009) consideran a la PPN y su variación estacional como servicios ecosistémicos (SE) intermedios clave, ya que determinan el nivel de provisión de varios SE finales como la producción de commodities, la captura de C, la protección del suelo, la purificación de agua, y la provisión de hábitat y refugio, entre otros (Viglizzo et al. 2011). A partir de conocer los cambios en la productividad primaria derivados de las distintas intervenciones humanas es posible estimar el sentido del cambio en el nivel de provisión de varios otros servicios ecosistémicos derivados (Verón et al. 2011). En sistemas dominados por herbáceas, los métodos tradicionales de estimación de la productividad primaria neta aérea (PPNA) se basan en cortes de biomasa (Sala and Austin 2000). En sistemas con un componente leñoso importante, la estimación suele realizarse a través de la recolección de las hojas, ramas y frutos caídos (Prause et al. 2003). Estos métodos resultan costosos en términos de tiempo y esfuerzo y, al tratarse de mediciones puntuales, las extrapolaciones a escala regional presentan serias dificultades (Baeza et al. 2011).

El modelo de eficiencia en el uso de la radiación (Monteith 1972) constituye una aproximación conceptualmente sólida que permite estimar la PPNA a partir de datos provistos por sensores remotos a bordo de satélites de manera relativamente sencilla y confiable para muchos ecosistemas (Paruelo and Vallejos 2013). Fue originalmente desarrollado para cultivos, aunque se los usó ampliamente para estimar la productividad de pastizales (e.g., Paruelo et al. 1997; Seaquist et al. 2003; Piñeiro et al. 2006; Grigera et al. 2007) y de bosques y forestaciones (e.g., Goetz and Prince 1996; Veroustraete 1996; Battaglia and Sands 1997; Olofsson et al. 2007). Dicho modelo establece que la PPNA de una cubierta vegetal está determinada por la radiación fotosintéticamente activa incidente (RFAi), por la fracción de esa radiación que es interceptada por los tejidos verdes (fRFA) y por la eficiencia de uso de la radiación (EUR) para convertir en tejidos vegetales. Los datos de reflectancia de la superficie registrados por sensores remotos a bordo de satélites permiten calcular índices que estiman la fRFA (Sellers et al. 1992; Gower et al. 1999; Pettorelli et al. 2005); el Índice de Vegetación Normalizado (IVN o NDVI, por sus siglas en inglés) es el que se usa más comúnmente. Se calcula como: IVN=(Rir-Rr)/ ( $\operatorname{Rir}+\mathrm{Rr})$, donde Rir y Rr son las reflectancias (proporción de la radiación incidente que es reflejada) de la superficie en la longitud del infrarrojo cercano y el rojo del espectro electromagnético, respectivamente. En sistemas en donde coexisten plantas herbáceas y leñosas se puede descomponer la señal de los componentes herbáceos y leñosos a partir de una serie temporal de datos espectrales (Lu et al. 2003). Esta técnica fue utilizada con éxito por Blanco et al. (2016) en bosques secos rolados de la provincia de La Rioja. La EUR, por su parte, varía entre especies y comunidades vegetales y ante modificaciones en las condiciones ambientales. Sin embargo, en diversos estudios se la considera constante en el tiempo y variable sólo según el tipo de cobertura, debido a que la radiación absorbida por la vegetación (RFAA) presenta una variación considerablemente mayor y es estimable con mayor precisión, dada la mayor facilidad para su medición (Ruimy et al. 1994; Grigera et al. 2007).

La región fitogeográfica del Chaco (Morello et al. 2012) se extiende por el norte de la Argentina y, junto con las Yungas, representan $\sim 81 \%$ de todos los bosques nativos del país (SAyDS 2005). El Chaco Semiárido es la principal subregión y se extiende mayormente por las provincias de Salta, Santiago del Estero, Chaco y Formosa. Aún conserva grandes extensiones de bosque no transformadas a agricultura, aunque desde principio de los años setenta experimentó un proceso de desmonte acelerado (Grau et al. 2005; 2008). Las tasas de desmonte tuvieron una tendencia creciente entre 1976 y 2012 (Volante et al. 2016), con valores hasta tres veces superiores que en los años ochenta (Piquer et al. 2015), lo que implicó la eliminación de casi 11.5 millones de hectáreas de bosque. El cultivo de soja es el principal impulsor de estos cambios (Gasparri and Grau 2009); en los últimos años pasó a representar entre 50 y $60 \%$ del total del área sembrada en la región. La ganadería intensiva de cría y engorde vacuno completa el mosaico del paisaje agropecuario de la región, actividad que se encuentra en franco crecimiento (Caruso et al. 2012). El proceso expansivo de la ganadería está asociado con el reemplazo del monte nativo por pasturas 
tropicales (principalmente de Gatton Panic, Megathyrsus maximus var. Gatton), a través del desmonte total o selectivo (sistemas silvopastoriles) (Caruso et al. 2012). En el primer caso, se elimina toda la cubierta forestal, mientras que en el segundo se realiza tala selectiva o desbajerado, dejando en pie los árboles de mayor porte. Este uso del suelo ha tomado impulso en los últimos años, motivado por la flexibilización por parte de algunas provincias de los criterios de uso y conservación definidos dentro de la categoría II (Amarillo) en la Ley de Ordenamiento Territorial de Bosques Nativos (Ley N ${ }^{\circ} 26331$ ) (García Collazo et al. 2013). En esta categoría solo se permite realizar un aprovechamiento sostenible del bosque que no implique tareas de desmonte; sin embargo, las provincias de Chaco y Santiago del Estero permitieron la implementación de sistemas silvopastoriles como una alternativa productiva (Chiossone et al. 2014). No obstante, en la mayoría de los casos, su implementación conlleva a una intensidad elevada de remoción de biomasa leñosa, lo que altera no sólo la estructura del bosque, sino también la reposición del estrato arbóreo (Casar 2016; Aguiar et al. 2018).

Trabajos previos en la región del Chaco Semiárido utilizan índices espectrales de vegetación como subrogado de la PPN (Paruelo et al. 2011) con el fin de evaluar el impacto del desmonte y de los cambios en el uso (Huykman 2011; Moreno 2012; Volante et al. 2012; Baldi et al. 2013; Staiano 2016; Basualdo et al. 2019). En todos los casos se concluye que tanto los cultivos como las pasturas incrementan la estacionalidad de la PPN respecto de los bosques nativos. Sin embargo, arriban a resultados opuestos en cuanto a la PPN total y ninguno puede concluir acerca del impacto de la transformación del tipo de cobertura sobre cuanta PPN se pierde o se gana. Además, sus resultados se encuentran circunscriptos a lo que sucede con la parte aérea sin considerar los cambios que ocurren en la porción subterránea, que suele variar entre tipos fisonómicos de vegetación. Así, los árboles tienden a destinar una fracción menor de la productividad primaria a estructuras subterráneas que los pastos. Mientras que la relación entre la biomasa aérea y la subterránea en bosques semiáridos es cercana a 3:1, en pasturas tropicales la relación suele ser inferior a 1:2 (Cairns et al. 1997; Jackson et al. 1996; Jobbágy and Jackson 2000; Mokany et al. 2006), y en los cultivos es superior a 7:1 (Miralles et al. 1997; Cárcova 1998).
El objetivo de este trabajo fue cuantificar el efecto de la transformación en el uso y cobertura del suelo sobre la PPN y su variación estacional. En particular, se evaluó el cambio, respecto del bosque original, de la PPN en sistemas agrícolas y silvopastoriles con un componente leñoso variable. Nuestra hipótesis sugiere que la conversión de la vegetación leñosa natural en pasturas y cultivos agrícolas reduce la productividad anual, ya que estos usos del suelo presentan una mayor estacionalidad en la actividad fotosintética (Volante et al. 2012). De esta manera, si bien las pasturas y cultivos agrícolas podrían alcanzar una mayor productividad, la misma se encuentra acotada a un período del año, identificando momentos de muy baja o nula productividad. Por otro lado, suponemos que la presencia de especies leñosas en los distintos usos del suelo analizados reduce la variación interanual de la productividad. Debido a que la vegetación leñosa presenta una mayor profundidad de exploración de raíces que los cultivos y las pasturas (Jackson et al. 1996), esto le permitiría utilizar el agua almacenada en la profundidad del suelo en años de baja disponibilidad hídrica.

\section{MATERIALES Y MÉTODOS}

\section{Área de estudio}

El área de estudio se localizó en el Departamento de Anta, al sudeste de la provincia de Salta, que limita con las provincias de Chaco y Santiago del Estero (Figura 1). Se seleccionó este departamento como área representativa de la región, dado que incluye todas las condiciones de usos abordadas en este trabajo (ver Materiales y Métodos, Identificación de usos del suelo). Tiene una superficie aproximada de 22160 $\mathrm{km}^{2}$, de los cuales más del $87 \%$ pertenecen al Chaco Semiárido. Presenta un gradiente de precipitación entre 600 y $800 \mathrm{~mm} /$ año en sentido sureste-noroeste (Figura 1) y una temperatura media anual entre 20 y $25{ }^{\circ} \mathrm{C}$, característico de un clima subtropical. Los bosques xerofíticos son la vegetación típica de esta región, que se encuentra dominada por especies caducifolias y semicaducifolias adaptadas a grandes fluctuaciones en la disponibilidad de agua y a variaciones estacionales en la temperatura. El quebracho colorado santiagueño (Schinopsis lorentzii), quebracho colorado chaqueño (Schinopsis balansae), quebracho blanco (Aspidosperma quebracho-blanco), guayacán (Caesalpinia 

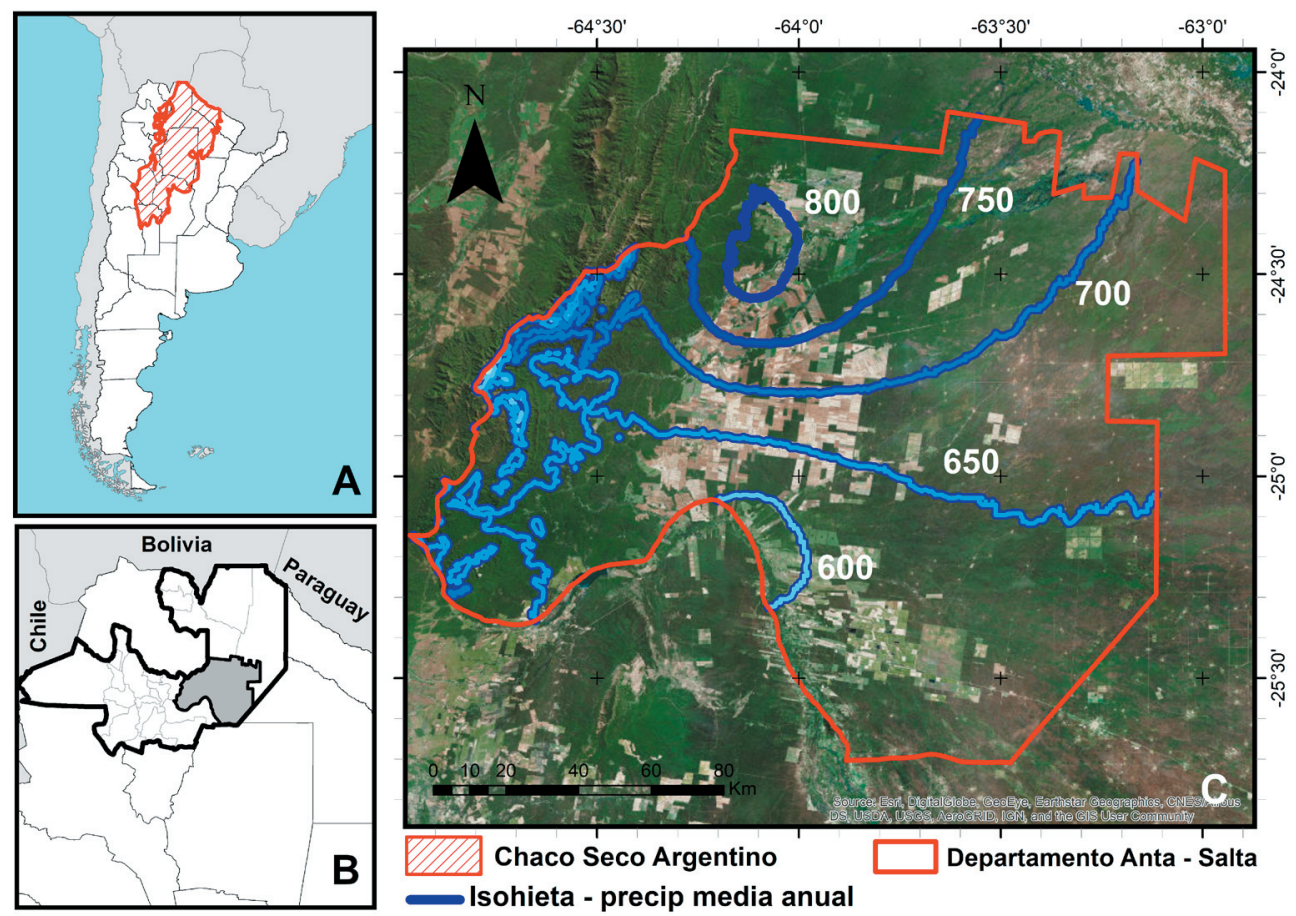

Figura 1. El área de estudio se encuentra localizada en la subregión del Chaco Semiárido, perteneciente al Chaco Seco Argentino (a). Específicamente, las estimaciones se realizaron en el Departamento de Anta, provincia de Salta, Argentina (b y c).

Figure 1. The study area is located in the sub-region of the semi-arid Chaco, belonging to the Dry Argentinian Chaco (a). Specifically, the estimates were made in the Department of Anta, province of Salta, Argentina (b and c).

paraguariensis),mistol(Ziziphusmistolyvarias especies del género Prosopis son los arboles más dominantes (Vargas Gil and Vorano 1985). También se identifican pastizales, sabanas arboladas abiertas y matorrales, los cuales se desarrollan en los paleocauces colmatados de los ríos principales (Morello et al. 2012). El mosaico de vegetación original se completa con áreas con vegetación arbustiva y arbórea semiabierta a semicerrada correspondiente a bosques secundarios xéricos originados a partir del bosque nativo por acción humana mediante el fuego y el uso ganadero excesivo (Morello et al. 2012).

La expansión de la frontera agrícola determinó la pérdida de extensas áreas de la vegetación natural, impulsada principalmente por la expansión del cultivo de soja (Paruelo et al. 2005; Gasparri et al. 2013; Fehlenberg et al. 2017). Sin embargo, buena parte del área también resulta apta para cultivos de maíz y trigo, que crecieron en superficie durante los últimos años (Baumann et al. 2017). La agricultura se caracteriza por ser de secano (con excepción de los sitios localizados en las inmediaciones del Río Juramento, en la porción sudeste del Departamento de Anta) y bajo siembra directa. La ganadería extensiva también ganó impulso en los últimos años, lo que promovió el reemplazo del bosque nativo por pasturas megatérmicas exóticas (Gasparri et. al. 2013; Volante et al. 2016). El Gatton Panic (Megathyrsus maximus var. Gatton) posee una excelente adaptación a los suelos y clima de la región y se ha convertido, desde hace años, en el principal recurso forrajero, debido principalmente a su alta productividad, a su fácil implantación y su sencillo manejo (Chiossone et al. 2014). El reemplazo de la cubierta forestal arbórea puede ser total o parcial, sistema comúnmente denominado "silvopastoril" (Caruso et al. 2012). En ellos, se utilizan rolos para remover parcialmente la vegetación leñosa dejando en pie solo los árboles de mayor porte $\mathrm{y}$, al mismo tiempo, sembrar semillas de pastos megatérmicos (Kunst et al. 2014).

\section{Identificación de usos del suelo}

Los tipos de uso del suelo incluyeron el bosque nativo, las pasturas megatérmicas puras y con presencia de árboles dispersos (sistemas silvopastoriles), y distintos cultivos agrícolas (trigo, soja y maíz). La identificación 
de estos usos se realizó mediante la utilización de una clasificación supervisada de usos del suelo del Departamento de Anta correspondiente a la campaña 2009-2010 (Bagnato et al. 2015) (Material Suplementario, Figura 1), del producto MOD44B V051 de Campos Continuos de Vegetación (VCF, por sus siglas en inglés) de MODIS (Material Suplementario, Figura 2), del Primer Inventario de Bosques Nativos (SAyDS 2005) (Material Suplementario, Figura 3), de una capa vectorial con información de los desmontes ocurridos en el NOA entre 1976 y 2015 (Vallejos et al. 2015) (Material Suplementario, Figura 4), y de información brindada por productores locales (Material Suplementario, Figura 5). La clasificación permitió discriminar entre pasturas, cultivos anuales y cultivos perennes con una fiabilidad global del 95\% (Material Suplementario, Tabla 1). Posteriormente, se utilizó el producto VCF de MODIS para diferenciar pixeles con distinta proporción de cobertura de árboles en los lotes clasificados como pasturas. Este producto posee una resolución espacial de $250 \mathrm{~m}$ y se genera a partir de la utilización de compuestos quincenales de reflectancia y temperatura superficial de MODIS. De esta manera, se buscó discriminar las pasturas puras sembradas luego del desmonte total, de aquellos sistemas que, producto del desmonte parcial y el uso de rolos, dejan en pie una cobertura variable de árboles. Así, se clasificaron los pixeles MODIS en 6 categorías: pasturas $(<1 \%)$, y sistemas silvopastoriles con distinta cobertura de árboles (entre $1-5 \%$, entre $5-10 \%$, entre $10-15 \%$, entre $15-20 \%$ y entre $20-$ $25 \%$ ) (Material Suplementario, Figura 2). Los sistemas silvopastoriles con una cobertura arbórea superior al 25\% no fueron incluidos en el análisis, debido a la reducida cantidad de pixeles registrados. Para el caso del bosque, se utilizó la capa vectorial del Primer Inventario Nacional de Bosques Nativos (SAyDS 2005) (Material Suplementario, Figura 3) y una capa vectorial de los desmontes registrados en la región (Vallejos et al. 2015) (Material Suplementario, Figura 4). Se seleccionaron todos los pixeles que no fueron desmontados hasta el año 2011 y que pertenecían a las categorías "Quebrachal típico" y "Quebrachal alto" (Nivel 3 del Inventario), evitando la inclusión de quebrachales degradados, pastizales naturales y antrópicos, matorrales y estepas arbustivas herbáceas (Material Suplementario, Figura 3).

En cuanto a los cultivos agrícolas, se recurrió a información provista por productores locales
(Material Suplementario, Figura 5). Si bien la clasificación de usos del suelo desarrollada por Bagnato et al. (2015) permitió identificar de manera muy precisa los cultivos anuales, la misma presentó una baja resolución conceptual (es decir, no fue capaz de distinguir entre tipos de cultivos, ya sean de invierno o de verano), y un acotado rango temporal (circunscripta a la campaña 2009-2010). Estas limitaciones dificultaron su uso para la caracterización de la PPN de los sistemas agrícolas. Por lo tanto, se tomaron como referencia 102 lotes agrícolas en donde productores locales brindaron información del tipo de uso anual y de las rotaciones realizadas en cada lote durante el período 2001-2010. Estos lotes fueron digitalizados utilizando imágenes de Google Earth de alta resolución espacial.

En total, se seleccionaron entre 73785 y 101733 pixeles MODIS (Tabla 1), considerando sólo los pixeles puros que estaban completamente incluidos en los límites de cada uso o cobertura del suelo. El rango de variación en la cantidad de pixeles seleccionados (Tabla 1) responde a la inclusión paulatina de lotes que fueron desmontados en algún momento durante el período 2001-2010. Así, se relevó una superficie entre 396 y 545 mil hectáreas del Departamento de Anta, considerando ambos extremos del gradiente de precipitación (Figura 1). Se consideró que no hubo transformación o reconversión entre tipos de

Tabla 1. Pixeles seleccionados para los principales usos del suelo del Departamento de Anta, Salta. En el caso de las pasturas y los sistemas silvopastoriles, se indica un rango de pixeles seleccionados. Dado que muchos lotes fueron desmontados durante el período 2001-2010 (información cronológica obtenida de Vallejos et al. 2015), la cantidad de pixeles seleccionada al final del período (número de la derecha) fue superior que la cantidad de pixeles seleccionados al inicio (número de la izquierda).

Table 1. Pixels selected for the main land uses of the Department of Anta, Salta. In the case of pastures and silvopastoral systems, a range of selected pixels is indicated. Given that many plots were clearing during the period 2001-2010 (chronological information obtained from Vallejos et al. [2015]), the number of pixels selected at the end of the period (number on the right) was greater than the number of pixels selected at the beginning (number on the left).

\begin{tabular}{ll}
\hline & Pixeles seleccionados \\
\hline Pastura & $7985-23109$ \\
Silvopastoril (5\%) & $2005-4802$ \\
Silvopastoril $(10 \%)$ & $3034-8901$ \\
Silvopastoril $(15 \%)$ & $597-3179$ \\
Silvopastoril $(20 \%)$ & $173-1398$ \\
Silvopastoril $(25 \%)$ & $22-375$ \\
Bosque & 57600 \\
Cultivos agrícolas & $2369(102$ lotes $)$ \\
Total & $73785-101733$ \\
\hline
\end{tabular}


uso del suelo productivos, es decir, que un pixel desmontado en 2003 y clasificado como pastura en la campaña 2009-2010, mantuvo siempre ese uso a lo largo del período. Si bien el Gatton Panic (Megathyrsus maximus var. Gatton) es el recurso más difundido en la región, también se existen otros tipos de pasturas como Green Panic (Panicum máximum var. Green), Grama Rhodes (Chloris gayana) y Buffel Grass (Cenchrus ciliaris), que se implantan en la región en mucha menor proporción (De León 2004; Riffel and Elizalde 2008; Ávila et al. 2014). Debido a esto, hemos identificado todos los recursos forrajeros como pasturas megatérmicas. Todas las operaciones se realizaron mediante el uso de QGIS 2.18.

\section{Estimación de la PPN}

La estimación de la PPNA de los distintos usos del suelo se realizó a partir del modelo de eficiencia en el uso de la radiación propuesto por Monteith (1972), y de información proveniente de sensores remotos a bordo de satélites para la estimación de la radiación fotosintéticamente activa absorbida (RFAA) de las distintas coberturas del suelo. Dicho modelo establece que la PPNA de una cubierta vegetal es proporcional a la radiación fotosintéticamente activa incidente (RFAi), a la fracción de esa radiación que es interceptada por los tejidos verdes (fRFA), y a la eficiencia de conversión (EUR) (Ecuación 1).

$$
\begin{aligned}
& \text { PPNA }=\text { fRFA * RFAi }\left(\text { MJ.m. }{ }^{-2} \cdot \mathrm{t}^{-1}\right) * \text { EUR }(\mathrm{g} \\
& \text { MS/MJ) } \\
& (\text { Ecuación 1) }
\end{aligned}
$$

La fRFA se estimó a partir del IVN, el cual es provisto por diversos sensores remotos a bordo de satélites, entre los que se destaca el MODIS por su adecuada resolución espacial y temporal. Este sensor, a bordo del satélite TERRA perteneciente a la NASA, provee una estimación de la intercepción de radiación por parte de la vegetación cada 16 días y para 5.4 ha de superficie desde el año 2000 . Específicamente, se utilizó información del período 2001-2010 correspondiente al producto MOD13Q1 para la escena h12v11, que cubre completamente la región bajo estudio. Se aplicó un filtro de calidad a las imágenes de IVN con el fin de descastar todos aquellos pixeles que presentaban nubes, sombras y / o aerosoles en la atmósfera, mediante el uso de la información de calidad disponible en la banda adicional QA. Los valores filtrados fueron reemplazados por el valor de IVN promedio correspondiente a la imagen inmediatamente anterior y posterior. La información espectral a escala quincenal fue mensualizada, prorrateando el valor de cada banda en función de la cantidad de días que integraba dentro del mes. La fRFA se estimó como una función lineal del IVN proveniente de las imágenes MODIS, diferencialmente según el tipo fisonómico de vegetación. Para el caso de los cultivos agrícolas, pasturas megatérmicas y componentes herbáceos de los sistemas silvopastoriles, se utilizó una parametrización generada a partir de cultivos de trigo (fRFA $=1.515^{*} \mathrm{IVN}-0.33$ ) (Grigera and Oesterheld 2006). Para el caso del bosque y los componentes leñosos de los sistemas silvopastoriles, se utilizó una parametrización generada experimentalmente para una especie leñosa (fRFA $=1.375^{*} \mathrm{IVN}-0.1831$ ) (Facio 2014). Los valores de fRFA inferiores a 0 y superiores a 0.95 fueron igualados a esos valores, respectivamente.

Se aplicó la metodología de Lu et al. (2003) con el fin de descomponer los valores originales de IVN en sus respectivos componentes herbáceos y leñosos y estimar, en los sistemas silvopastoriles, el aporte de cada uno de ellos. La metodología parte de la asunción de que la vegetación herbácea y leñosa presentan un comportamiento fenológico distinto debido, entre otras cosas, a la respuesta diferencial que poseen ambos tipos de vegetación a factores ambientales, como la precipitación y la temperatura. Mientras que la vegetación leñosa tiene una débil onda fenológica anual que fluctúa en respuesta a la variabilidad climática interanual, la vegetación herbácea presenta una fuerte variación fenológica anual, con cambios en la amplitud de año en año, que responden a la variabilidad climática. Según este método, es posible caracterizar la fenología de ambos componentes mediante el cálculo de distintas variables obtenidas a partir del análisis de una serie temporal de IVN. Al mismo tiempo, supone que la contribución de los componentes leñosos y herbáceos al IVN total es aditiva, dado que supone que en los espacios cubiertos por el componente leñoso no hay presencia de herbáceas y viceversa, es decir, no contempla la presencia de componentes herbáceos bajo la copa de los árboles. Por esta razón, no se estimó la productividad de los pastos ubicados debajo de la copa arbórea en estos sistemas. El procesamiento de las imágenes se realizó en Python y se utilizó la función STL (Seasonal Descomposition of Times Series by LOESS) del paquete stats de R. El STL (Cleveland 
et al. 1990) es un procedimiento de filtrado basado en una secuencia de regresiones suaves ponderadas localmente que permite desagregar una serie de tiempo de IVN en tres componentes: tendencia, estacionalidad y residual y, a partir de ellos, estimar diferentes variables asociadas a atributos fenológicos de la vegetación herbácea y leñosa.

La radiación total incidente varía principalmente con la latitud, el momento del año y las condiciones atmosféricas, por lo que resulta necesario contar con una información espacialmente explícita y con una adecuada temporalidad, mejor que la brindada por las escasas estaciones meteorológicas de la región. Se utilizó el producto de Radiación Solar Global Media Mensual en W/ $\mathrm{m}^{2}$ (Ceballos and Macedo 2014), desarrollado por el Instituto Nacional de Pesquisas Espaciais (INPE). Este producto, basado en imágenes satelitales del sensor GOES, posee una resolución espacial de $0.04^{\circ}$, una extensión casi completa de Sudamérica, y se encuentra disponible a partir de enero de 2000. La RFA es una porción del total del espectro electromagnético (400 a 700 $\mathrm{nm})$ que está directamente relacionada con la producción fotosintética de las plantas (Ting and Giacomelli 1986), y se estimó como el $48 \%$ de la radiación solar total incidente (Blackburn and Proctor 1983). Se aplicaron los cálculos necesarios para la conversión de unidades de $\mathrm{W} / \mathrm{m}^{2}$ a MJ.me ${ }^{-2} \mathrm{mes}^{-1}$.

La EUR de cultivos agrícolas y del bosque se estimó a partir de valores obtenidos de la literatura científica, mientras que para pasturas megatérmicas se utilizaron valores estimados a campo para la región (Baldassini et al. 2018). Para el caso de los cultivos agrícolas, se utilizó una EUR de 1.095 g MS.MJ ${ }^{-1} . \mathrm{m}^{2}, 1.86$ g MS.MJ ${ }^{-1} . \mathrm{m}^{2}$ y $0.86 \mathrm{~g}$ MS.MJ ${ }^{-1} \cdot \mathrm{m}^{2}$ para trigo (Calderini et al. 1997), maíz (Otegui et al. 1995) y soja (Leadley et al. 1990; Muchow et al. 1993), respectivamente. El valor de EUR del bosque surgió de ajustar, en términos relativos, un valor a escala local en función de datos promedio de EUR obtenidos de modelos globales (Matthews 1983; Ruimy et al. 1994; Field et al. 1995). Se calculó una relación entre la EUR promedio de bosques secos $\left(0.76 \mathrm{~g} \mathrm{MS} . \mathrm{MJ}^{-1} . \mathrm{m}^{-2}\right)$ y pastizales $(1.04 \mathrm{~g}$ MS.MJ ${ }^{-1} \cdot \mathrm{m}^{-2}$ equivalente a 0.73 . Considerando esa relación y un valor de EUR de $0.7 \mathrm{~g}$ MS.MJ${ }^{1} . \mathrm{m}^{-2}$ obtenido por Oyarzabal et al. (2011) para un pastizal de referencia del Río de la Plata, se estimó la EUR del bosque chaqueño en $0.51 \mathrm{~g}$ MS.MJ ${ }^{-1} \cdot \mathrm{m}^{-2}$. Para las pasturas megatérmicas se utilizó una EUR variable para cuatro momentos de la estación de crecimiento (primavera, verano, verano tardío y otoño) determinada para pasturas de Gatton Panic (Megathyrsusmaximusvar.Gatton)(Baldassini et al. 2018), siendo los valores de eficiencia entre 0.59 y $1.29 \mathrm{~g} \mathrm{MS}^{\mathrm{MJ}}{ }^{-1} . \mathrm{m}^{-2}$.

La PPNS y la PPN de los distintos usos del suelo se calcularon a partir de relaciones PPNS/PPN disponibles en la literatura científica. Se utilizó una relación PPNS/PPN de 0.295 para el bosque xerofítico, según lo reportado por Martínez-Irizar (2009) para los bosques secos de Chamela, México, que presentan similares condiciones climáticas que el Chaco (707 mm de precipitación media anual, $24.9^{\circ} \mathrm{C}$ de temperatura media anual y 68 meses de sequía). Para los cultivos agrícolas, se utilizaron los valores promedio reportados por Guerschman (2005), a partir de distintos experimentos desarrollados en Argentina: 0.127 para maíz, 0.14 para trigo y 0.182 para soja. Para el caso de las pasturas megatérmicas, se utilizó una relación de 0.61 obtenida a través del promedio de las relaciones reportadas para diferentes especies $\mathrm{C}_{4}$ (Hui and Jackson 2005).

\section{Análisis estadístico}

Se analizó la integral anual y la dinámica estacional promedio de la PPNA, la PPNS, y la PPN obtenidos para cada uno de los usos del suelo. A su vez, se calculó la variación interanual para el período 2001-2010, tanto a escala anual como mensual. Cada campaña (de agosto a julio) fue considerada como una repetición $(n=9)$, a partir de las cuales se aplicaron modelos lineales mixtos, considerando el uso del suelo como factor fijo. Se incluyó la correlación temporal entre las campañas y la existencia de heteroscedasticidad entre usos, y se utilizó el método LSD Fisher para la comparación múltiple entre medias. Los análisis fueron realizados utilizando el paquete INFOSTAT (INFOSTAT 2008). Se asumió significación estadística con un valor- $\mathrm{p}<0.05$.

\section{Resultados}

Los distintos usos del suelo presentaron diferencias significativas en la productividad total, y fueron más importantes en la porción subterránea que en la parte aérea (Figura 2 y Tabla 2). La PPNA promedio del doble cultivo trigo-maíz fue significativamente superior a la del bosque (14946 vs. $11063 \mathrm{~kg}$ MS.ha ${ }^{-1} \cdot$ año $^{-1}$ ), mientras que el cultivo simple de maíz, con 


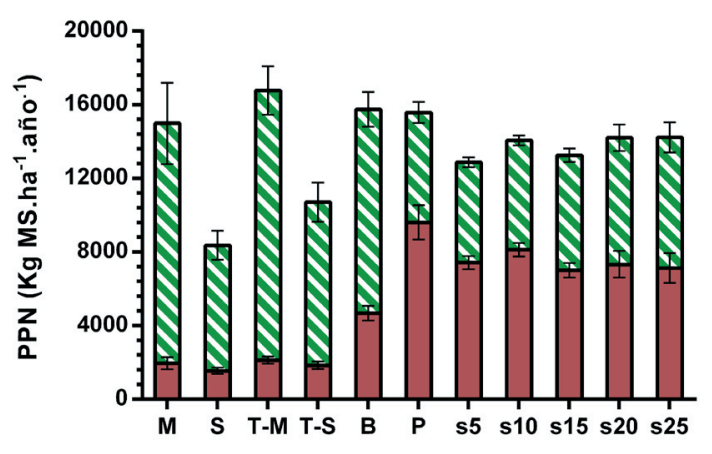

Figura 2. Productividad primaria neta (PPN) promedio anual (en kg MS.ha- ${ }^{-1}$. ño ${ }^{-1}$ ) para el período 2001-2010 de los principales usos del suelo ubicados en el Departamento de Anta, Salta: Maíz (M), Soja (S), Trigo-Maíz (T-M), Trigo-Soja (T-S), Bosque (B), Pasturas (P), y Sistemas silvopastoriles con cobertura arbórea entre 5 y $25 \%$ (s5, s10, s15, s20 y s25). La productividad primaria neta aérea (PPNA) promedio anual se indica en color verde y blanco, mientras que la productividad primaria neta subterránea (PPNS) se encuentra indicada en color marrón. Las líneas sobre las barras indican el desvío estándar interanual.

Figure 2. Average annual net primary production (NPP) (in $\mathrm{kg} \mathrm{DM} . \mathrm{ha}^{-1} \cdot \mathrm{yr}^{-1}$ ) for the period 2001-2010 of the main land uses located in the Department of Anta, Salta: Maize (M), Soybean (S), Wheat-Maize (T-M), Wheat-Soybean (T-S), Forest (B), Pastures (P), and Silvopastoral Systems with tree cover between 5 and $25 \%$ (s5, s10, s15, s20 and s25). The average annual aboveground net primary production (ANPP) is indicated in green and white, while the belowground net primary production (BNPP) is indicated in brown. The lines on the bars indicate the standard interannual deviation.

un valor promedio de $12729 \mathrm{~kg} \mathrm{MS} \mathrm{ha}^{-1}$.año ${ }^{1}$, no difirió significativamente del bosque (Figura 2 y Tabla 2). El resto de los usos del suelo (trigo-soja, soja de primera, pasturas y sistemas silvopastoriles) presentaron una
PPNA promedio significativamente inferior a la del cultivo de maíz de primera, al doble cultivo trigo-maíz y al bosque (Figura 2 y Tabla 2). Entre éstos, el doble cultivo trigo-soja mostró una PPNA significativamente superior al resto de los usos $\left(8882 \mathrm{~kg} \mathrm{MS} \mathrm{ha}^{-1} \cdot \mathrm{año}^{-1}\right)$, los cuales no difirieron de manera significativa entre sí (Figura 2 y Tabla 2). Los sistemas silvopastoriles de menor cobertura arbórea (5\%) fueron el uso menos productivo, con un

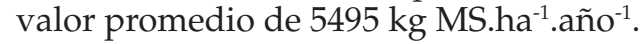

La PPNS mostró un patrón casi opuesto al observado con la PPNA. Mientras que las pasturas y los sistemas silvopastoriles fueron los usos más productivos, los diferentes cultivos agrícolas mostraron los niveles más bajos, observando valores intermedios para el bosque $\left(4675 \mathrm{~kg} \mathrm{MS} \mathrm{ha}^{-1}\right.$.año-1 ) (Figura 2 y Tabla 2). Las pasturas tuvieron una PPNS promedio de $9606 \mathrm{~kg} \mathrm{MS} \cdot \mathrm{ha}^{-1} \cdot \mathrm{año}^{-1}$, significativamente superior a la de los sistemas silvopastoriles, que mostraron valores entre 7011 y $8127 \mathrm{~kg}$ MS.ha ${ }^{-1} \cdot a n ̃ o^{-1}$. Entre los cultivos agrícolas, el trigo-maíz fue el uso más productivo (2132 $\left.\mathrm{kg} \mathrm{MS} \cdot \mathrm{ha}^{-1} \cdot \mathrm{año}^{-1}\right)$, mientras que la soja de primera mostró los valores más bajos (1548 kg MS.ha $\left.a^{-1} \cdot a^{-1} o^{-1}\right)$.

La PPN total, derivada de la suma de la PPNA y la PPNS, tuvo un patrón distinto debido a las diferencias observadas entre la parte aérea y subterránea entre los usos del suelo. Sin embargo, el doble cultivo trigomaíz fue el uso más productivo, con valores promedio de $16772 \mathrm{~kg} \mathrm{MS} \cdot \mathrm{ha}^{-1} \cdot \mathrm{año}^{-1}$, aunque no difirió significativamente del bosque (15748

Tabla 2. Prueba LSD Fisher de comparación múltiple entre medias de la productividad primaria neta (PPN), de la productividad primaria neta aérea (PPNA) y de la productividad primaria neta subterránea (PPNS) entre los distintos usos del suelo del Departamento de Anta, Salta.

Table 2. Fisher LSD test of multiple comparison between means of net primary production (PPN), aboveground net primary production (PPNA) and belowground net primary production (PPNS) among the different land uses of the Department of Anta, Salta.

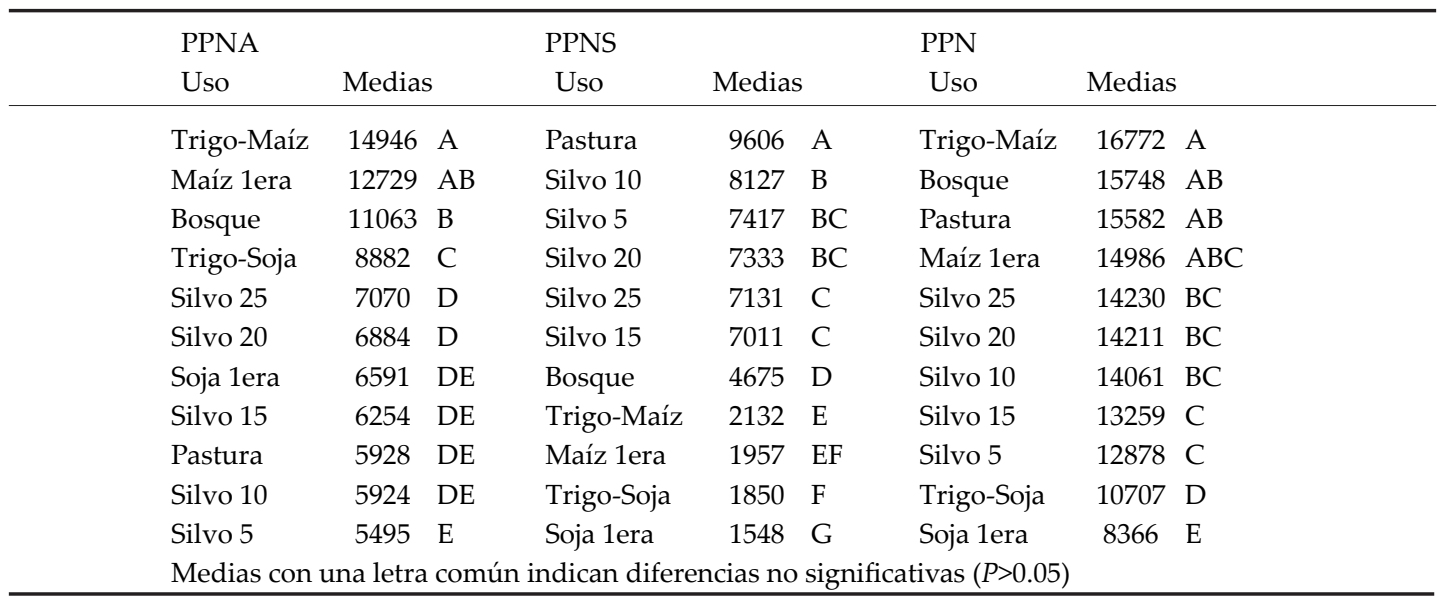


kg MS.ha-1 año-1 $^{-1}$, de las pasturas $(15582 \mathrm{~kg}$ MS.ha- $\left.{ }^{-1} \cdot a_{n} o^{-1}\right)$ y del cultivo simple de maíz (14986 kg MS.ha ${ }^{-1}$.año ${ }^{-1}$ ) (Figura 2 y Tabla 2). Los sistemas silvopastoriles, con valores entre 14230 y $12878 \mathrm{~kg} \mathrm{MS} \cdot$ ha $^{-1}$.año ${ }^{-1}$, difirieron significativamente con la PPN del bosque y de las pasturas sólo cuando presentaron una menor cobertura arbórea (5 y 15\%) (Figura 2 y Tabla 2). Por su parte, la menor productividad estuvo representada por el doble cultivo trigosoja y por la soja de primera, con valores promedio de 10707 y $8366 \mathrm{~kg}$ MS.ha- ${ }^{-1} \cdot \mathrm{año}^{-1}$, respectivamente (Figura 2 y Tabla 2).

En el caso particular de las pasturas y los sistemas silvopastoriles, no se observaron diferencias significativas en la PPN a lo largo del gradiente de cobertura arbórea (Figura 2 y Tabla 2). Sin embargo, al observar de manera separada la PPNA y la PPNS, se observaron diferencias significativas entre los diferentes sistemas en la mayor parte de las situaciones. Así, mientras que los sistemas con 20 y $25 \%$ de cobertura arbórea presentaron una PPNA significativamente diferente de los sistemas con 5\% de cobertura leñosa, los sistemas con $10 \%$ de cobertura arbórea presentaron una PPNS significativamente mayor que aquellos con una cobertura leñosa de 15 y $25 \%$. Por cada incremento unitario en la cobertura leñosa, la PPNA de los pastos se redujo, en promedio, $89 \mathrm{~kg} \mathrm{MS} \cdot \mathrm{ha}^{-1} \cdot \mathrm{año}^{-1}$, mientras que la PPNA leñosa se incrementó $135 \mathrm{~kg}$ MS.ha- ${ }^{-1} \cdot \mathrm{año}^{-1}$ (Figura 3). A su vez, la PPNS de los pastos se redujo $141 \mathrm{~kg} \mathrm{MS} \cdot \mathrm{ha}^{-1} \cdot \mathrm{año}^{-1}$, mientras que la PPNS leñosa aumentó $57 \mathrm{~kg}$ MS.ha- ${ }^{-1} \cdot \mathrm{año}^{-1}$ (Figura 3). Es decir, si bien el incremento de la cobertura leñosa redujo la productividad de los pastos en la parte aérea, las pérdidas fueron compensadas por un incremento en la productividad delos componentes leñosos. Por el contrario, la reducción en la productividad de los pastos en la parte subterránea no pudo ser compensada por el incremento en la productividad de los componentes leñosos (Figura 3). El balance en la parte subterránea no sólo fue negativo, sino que además fue superior, en términos reales, al balance de la porción aérea. Al no observarse diferencias significativas en la PPN, los datos sugerirían que las pasturas y los sistemas silvopastoriles no difieren en su productividad total, pero si en la alocación de esa productividad en la parte aérea y subterránea (Figura 3).

Todas las coberturas presentaron un claro patrón estacional en la PPN, con valores máximos durante el verano y valores mínimos durante el invierno (Figura 4). Tanto el bosque, como las pasturas y los sistemas silvopastoriles presentaron, en promedio, la máxima PPN durante el mes de enero, con valores cercanos a los 2000, 4900 y $3700 \mathrm{~kg}$ MS.ha ${ }^{-1} \cdot \mathrm{mes}^{-1}$, respectivamente. Sin embargo, las pasturas y los sistemas silvopastoriles concentraron su productividad entre los meses de diciembre y febrero, al producir entre 70 y el $80 \%$ y entre el 60 y $70 \%$ de registrado para todo el año, respectivamente (Figura 4). Por el contrario, si bien el bosque también tuvo una

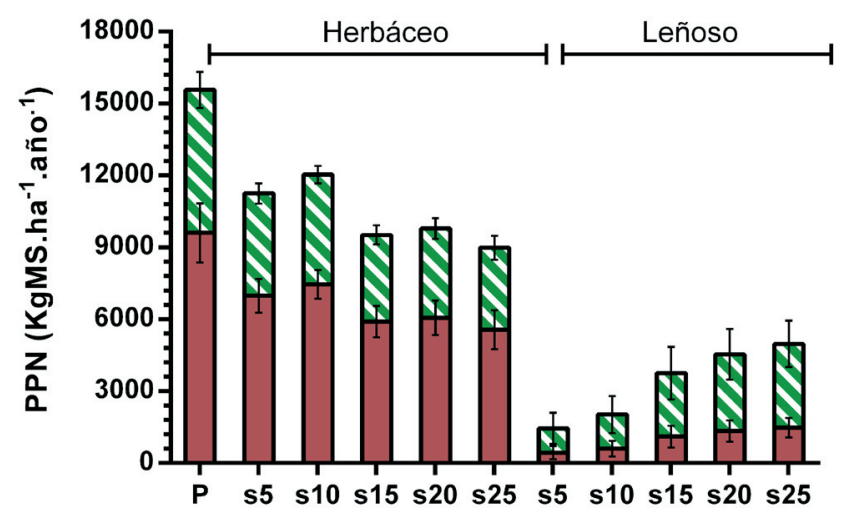

Figura 3. Productividad primaria neta (PPN) promedio anual (en kg MS.ha ${ }^{-1}$.año ${ }^{-1}$ ) para el período 2001-2010 de los componentes herbáceos y leñosos en pasturas megatérmicas $(\mathrm{P})$ y en sistemas silvopastoriles con cobertura arbórea entre 5 y $25 \%$ (s5, s10, s15, s20 y s25) en el departamento de Anta, Salta. La productividad primaria neta aérea (PPNA) se indica en color verde y blanco, mientras que la Productividad primaria neta subterránea (PPNS) se indica en color marrón. Las líneas sobre las barras indican el desvío estándar interanual.

Figure 3. Average annual net primary production (NPP) (in kg DM.ha-1. $\mathrm{yr}^{-1}$ ) for the period 2001-2010 of the herbaceous and woody components in tropical pastures (P) and in silvopastoral systems with tree cover between 5 and $25 \%$ (s5, s10, s15, s20 and s25) in the department of Anta, Salta. Aboveground net primary production (ANPP) is indicated in green and white, while belowground net primary production (BNPP) is indicated in brown. The lines on the bars indicate the standard interannual deviation. 
Bosque

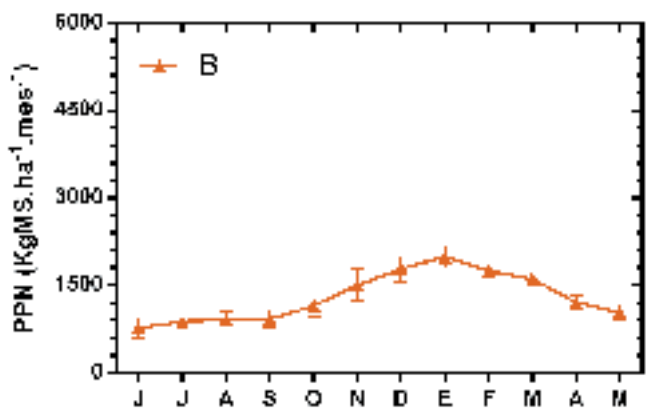

Pasturas

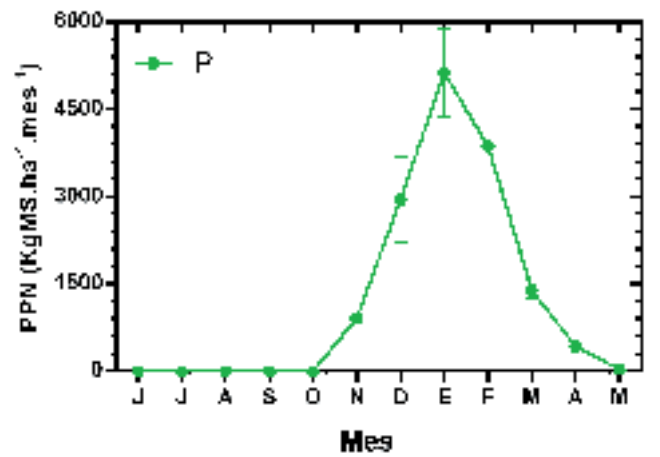

Cultivos agricolas

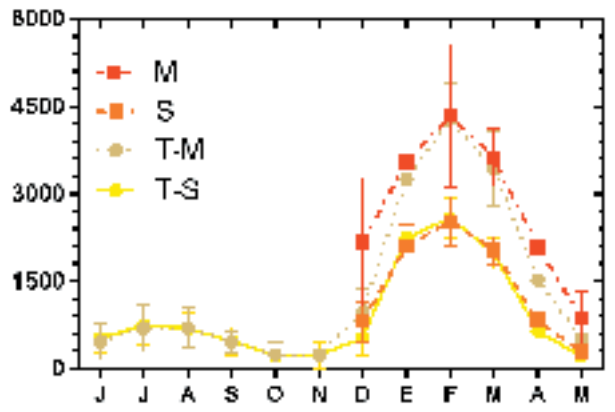

Silvopastoril

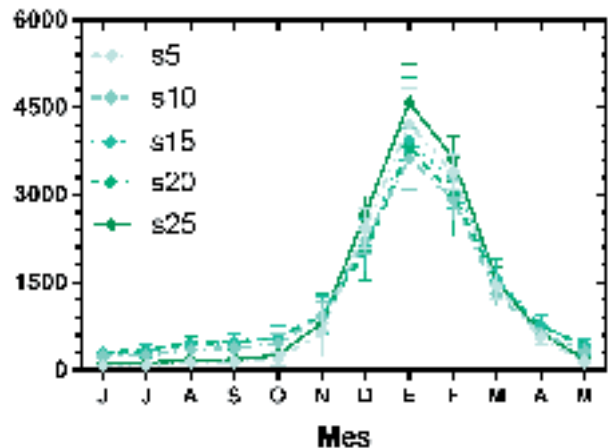

Figura 4. Productividad primaria neta (PPN) promedio mensual (en kg MS.ha- ${ }^{-1} \cdot \mathrm{mes}^{-1}$ ) para el período 2001-2010 de los principales usos del suelo del Departamento de Anta, Salta: Maíz (M), Soja (S), Trigo-Maíz (T-M), Trigo-Soja (T-S), Bosque, Pasturas y Sistemas silvopastoriles con cobertura arbórea entre 5 y 25\% (s5, s10, s15, s20 y s25). Las líneas verticales indican el desvío estándar interanual.

Figure 4. Average monthly net primary production (NPP) (in kg DM.ha ${ }^{-1} \cdot \mathrm{month}^{-1}$ ) for the period 2001-2010 of the main land uses of the Department of Anta, Salta: Maize (M), Soybean (S), Wheat-Maize (T-M), Wheat-Soybean (T-S), Forest, Pastures and Silvopastoral Systems with tree cover between 5 and 25\% (s5, s10, s15, s20 and s25). The vertical lines indicate the interannual standard deviation.

mayor productividad total durante el verano, la productividad estuvo más distribuida a lo largo del año, concentrando durante esos tres meses tan sólo el 35\% de la productividad anual (Figura 4). En el caso de los cultivos agrícolas de verano, la PPN máxima se registró en los meses de febrero (con excepción del maíz de primera, donde se observó en el mes de marzo), con valores cercanos a los 2500 $\mathrm{kg} \mathrm{MS} \cdot \mathrm{ha}^{-1} \cdot \mathrm{mes}^{-1}$ para el caso de la soja y de $4300 \mathrm{~kg} \mathrm{MS}$.ha ${ }^{-1}$. $\mathrm{mes}^{-1}$ para el maíz. El período productivo fue de diciembre a mayo en ambos casos (Figura 4). El cultivo de trigo mostró su máxima PPN durante el mes de agosto, con valores promedio cercanos a los $700 \mathrm{~kg}$ MS.ha ${ }^{1}$. $\mathrm{mes}^{-1}$ (Figura 4), y su período productivo se extendió de julio a noviembre. En cuanto a los valores mínimos mensuales, el bosque y los sistemas silvopastoriles mostraron la PPN más baja durante el mes de junio (Figura 4). Las pasturas no presentaron productividad durante el período junio-noviembre, y registraron sus menores valores de PPN al final del período de crecimiento (abril-

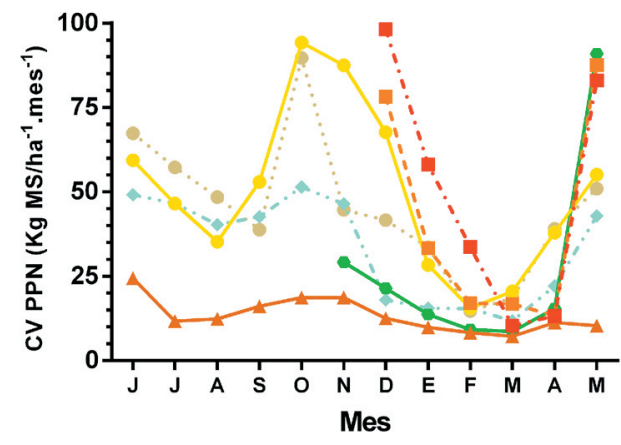

$-\mathrm{M}-\mathrm{E} \cdot \mathrm{S} \cdot \mathrm{T}-\mathrm{M} \rightarrow \mathrm{T}-\mathrm{S} \leadsto \mathrm{B} \rightarrow \mathrm{P} \rightarrow \mathrm{SP}$

Figura 5. Coeficiente de variación $(\mathrm{CV})$ interanual de la productividad primaria neta (PPN) mensual para el período 2001-2010 de los principales usos del suelo en el Departamento de Anta, Salta: Maíz (M), Soja (S), TrigoMaíz (T-M), Trigo-Soja (T-S), Bosque (B), Pasturas (P), y Sistemas silvopastoriles con cobertura arbórea entre 5 y $25 \%$ (SP).

Figure 5. Interannual variation coefficient (VC) of the monthly net primary production (PPN) for the period 2001-2010 of the main land uses in the Department of Anta, Salta: Maize (M), Soybean (S), Wheat-Maize (T-M), WheatSoya (T-S), Forest (B), Pastures (P), and Silvopastoral Systems with tree cover between 5 and $25 \%$ (SP). 
mayo) (Figura 4). Los cultivos de verano no presentaron productividad durante el período junio-diciembre, y en el mes de mayo se registró la menor PPN, mientras que en el cultivo de trigo el mínimo ocurrió durante el mes de noviembre (Figura 4).

El bosque presentó la menor variabilidad interanual de la PPN anual, con valores del CV en torno al 8\%. A su vez, junio fue el mes más variable entre años, con un valor promedio del 24\% (Figuras 4 y 5). Las pasturas presentaron una variabilidad del $10 \%$, siendo más variable durante el mes de mayo con valores cercanos al 90\% (Figuras 4 y 5). Los sistemas silvopastoriles fueron más variables entre años a medida que se incrementó la cobertura leñosa. Se observó una variabilidad interanual en torno al $5 \%$ para sistemas con menos de $15 \%$ de cobertura arbórea, mientras que aquellos con una cobertura leñosa superior al 20\% mostraron una variabilidad del 12\% (Figuras 4 y 5). Estos sistemas mostraron la mayor variabilidad entre años, en promedio, durante el mes de octubre, con valores cercanos al 50\% (Figuras 4 y 5). Los cultivos simples (soja y maíz de primera) mostraron una mayor variabilidad interanual que los doble cultivos (trigo-maíz y trigo-soja), con valores entre 12 y $17 \%$ y entre 9 y $12 \%$, respectivamente. En el caso de los cultivos simples, la mayor variabilidad se observó al inicio y al final del período de crecimiento (diciembre y mayo) con valores de 98 y $83 \%$ en el caso del maíz, y de 78 y $88 \%$ en el caso de la soja. En los que respecta a los dobles cultivos, octubre fue el mes más variable en ambos casos, con un CV de $94 \%$ en trigo-soja, y del $90 \%$ en el trigo maíz (Figuras 4 y 5).

\section{Discusión}

Los resultados observados difirieron de nuestra hipótesis planteada, ya que, si bien la PPN fue mayor en sistemas con una mayor presencia de especies leñosas, no se diferenciaron significativamente de las pasturas y de los cultivos de maíz y de trigo-maíz. Los sistemas silvopastoriles que presentaban una mayor cobertura de árboles tampoco difirieron con la productividad total del bosque, aunque sí lo hicieron con el doble cultivo trigo-maíz, mostrando una menor productividad. Los cultivos de soja y trigo-soja fueron los usos que menor PPN presentaron, y difirieron significativamente del resto de los usos analizados. Los similares valores observados entre el bosque y los distintos usos productivos en la productividad total, se asociaron a la partición diferencial de la productividad aérea y subterránea. Mientras que los cultivos presentaron una alta productividad aérea, mayor a la observada en las pasturas y los sistemas silvopastoriles, se observó lo contrario con respecto a la productividad subterránea, mostrando los bosques productividades intermedias en ambos casos. Sin embargo, si en los diferentes usos del suelo productivos (cultivos, pasturas y sistemas silvopastoriles) solo se considera la biomasa disponible después de la cosecha o el consumo ganadero, las contribuciones al sistema resultan inferiores que la vegetación leñosa original.

La PPN estimada para los distintos usos del suelo estuvo, en general, dentro del rango reportado por la bibliografía. En el caso del bosque, la PPN promedio fue de $15748 \mathrm{~kg}$ MS.ha-1.año-1, muy similar a lo estimado por Haberl et al. (2007) quien, a partir de una integración espacial de alta resolución de las mejores bases de datos globales disponibles, reportó una productividad total promedio de 15507 kg MS.ha-1 año $^{-1}$. Sin embargo, estos valores resultaron bastante más elevados que los reportados por Rueda et al. (2013), quien estimó la PPN de los sistemas leñosos del Chaco seco a partir de un modelo de regresión simple basado en datos de temperatura y precipitación media (Del Grosso et al. 2008). Según dicho modelo, la PPN promedio sería de 9590 kg MS.ha-1. año-1, resultando un 39\% inferior al estimado en este trabajo. En el caso de la PPNA se observa el mismo patrón, en donde a partir de las regresiones de Del Grosso et al. (2008) se obtienen estimaciones de productividad aérea en torno a los 6283 $\mathrm{kg}$ MS.ha ${ }^{-1} \cdot \mathrm{anno}^{-1}$, siendo un $43 \%$ inferior a los aquí estimados (11063 kg MS.ha ${ }^{-1} \cdot$ año $\left.^{-1}\right)$. En las pasturas, la PPNA promedio estimada fue de $5928 \mathrm{~kg}$ MS.ha ${ }^{-1}$.año ${ }^{-1}$, lo cual concuerda con los mencionados por otros autores. Pérez (2005) indica una producción entre 4500 a $7500 \mathrm{Kg}$ MS.ha-1 año $^{-1}$ para zonas de 550 a 750 $\mathrm{mm} / \mathrm{año}$, De León (2004) informa valores entre 4000 y $7500 \mathrm{~kg}$ MS.ha ${ }^{-1}$.año ${ }^{-1}$ en Santiago del Estero para zonas de 400 y 800 mm/año, respectivamente, y Chiossone et al. (2014) reporta una producción promedio de $8276 \mathrm{~kg}$ MS.ha-1.año-1 para sitios con una precipitación anual promedio de $800 \mathrm{~mm}$. Para el caso de los cultivos agrícolas, el uso de índices de cosecha (0.35 para trigo, 0.465 para maíz y 0.45 para soja [Cárcova et al. 2004]), permite estimar los rendimientos a partir de los valores de PPNA 
y compararlos con los valores promedio reportados por el Portal de Datos Abiertos (Secretaría de Agroindustria de Argentina) para el Departamento de Anta, Salta. Mientras que el rendimiento promedio estimado de maíz, soja y trigo para la región durante el período 2001-2010 fue de 5919, 2966 y $789 \mathrm{~kg}$ MS.ha ${ }^{-1} \cdot \mathrm{año}^{-1}$, respectivamente, el promedio de todo el Departamento reportado por la Secretaría para el mismo período fue de 4489 , 2583 y $1289 \mathrm{~kg}$ MS.ha- ${ }^{-1} \cdot \mathrm{año}^{-1}$.

En lo que respecta a las diferencias de productividad en la porción aérea entre los distintos usos del suelo, los cultivos de maíz y trigo-maíz presentaron una PPNA similar o superior que la del bosque, y fueron significativamente mayores que las productividades observadas para las pasturas puras, los sistemas silvopastoriles y los cultivos de soja. Sin embargo, estos resultados difirieron fuertemente de aquellos reportados por trabajos previos en el Chaco Semiárido que utilizan distintos índices espectrales de vegetación como proxy de la productividad primaria. Mientras que Huykman (2011), Volante et al. (2012) y Basualdo et al. (2019) observaron una mayor productividad en la vegetación natural que los cultivos o pasturas, Baldi et al. (2013) no encontraron diferencias significativas entre ambos grupos en transectas ubicadas a lo largo de un gradiente de estrés hídrico. Por el contrario, Staiano et al. (2016) observaron que los cultivos presentaron una mayor productividad que los bosques, siendo la soja, por sobre los cultivos de poroto y maíz y las pasturas, la que presentó la diferencia de mayor magnitud (10\% superior). Por otro lado, el maíz de primera y el doble cultivo trigo-maíz presentaron una PPNA significativamente mayor que el doble cultivo trigo-soja, la soja de primera y las pasturas megatérmicas. Esto concuerda con lo observado por Murray et al. (2016), donde el maíz produjo siempre una mayor cantidad de biomasa aérea con respecto a otras coberturas a lo largo de un gradiente de precipitación en el Chaco y el Espinal. Sin embargo, estos autores recomiendan al maíz como la única opción de cultivo en la región, sin ningún nicho oportuno para el desarrollo de pasturas y la vegetación natural basado únicamente en estimaciones de PPNA, sin considerar las diferencias existentes en la productividad subterránea. Por el contrario, estos resultados no concordaron con los reportados por Moreno (2012) y Staiano (2016), quienes prácticamente no encontraron diferencias en la productividad entre los distintos usos productivos (cultivos de soja, maíz, poroto y pasturas). Independientemente de los resultados obtenidos en cada uno de los trabajos reportados, ninguno de los autores ha podido definir con certeza cómo es el cambio de la productividad total en los diferentes usos del suelo.

La variación interanual de la productividad concordó con nuestra suposición inicial, resultando inferior en sistemas con presencia de especies leñosas. Esto podría estar asociado con el mayor desarrollo radical que poseen las coberturas leñosas en comparación con las distintas coberturas herbáceas, lo que les permitiría explorar el suelo a mayor profundidad y acceder al agua ubicada a mayor profundidad. Las herbáceas concentran entre el 80 y el $90 \%$ de las raíces en los primeros $30 \mathrm{~cm}$ del suelo, mientras que los árboles registran el $60 \%$ a esa profundidad, siendo la profundidad de enraizamiento de 1 y $2 \mathrm{~m}$, respectivamente (Jackson et al. 1996, en base al mapa global con resolución espacial de $1^{\circ}$ ). De esta manera, el bosque tendría mayor capacidad de amortiguación ante fluctuaciones climáticas respecto de los cultivos o pasturas (Volante et al. 2012). Si bien la variabilidad fue relativamente baja a escala anual, se observó un gran incremento a escala mensual, con una mayor variabilidad al inicio y al final de la estación de crecimiento. En los sistemas dominados por herbáceas, esto puede estar asociado al comienzo del período de lluvias hacia finales de la primavera, y a las bajas temperatura y heladas tempranas registradas hacia finales del otoño (Cornacchione and Molina 2008), respectivamente. Las pasturas megatérmicas comienzan a crecer con una precipitación acumulada de $30-40 \mathrm{~mm}$ y por encima de los $150{ }^{\circ} \mathrm{C} /$ día (Kunst et al. 2014), mientras que la fecha de siembra de los cultivos está asociada a la ocurrencia de las primeras precipitaciones (Volante et al. 2001). En soja y maíz de secano, la actividad de siembra se observa desde mediados de noviembre a diciembre (Galván 2002), para cosechar desde la primera quincena de abril (Volante et al. 2001). Cuando se dispone de buenas condiciones de humedad en el suelo durante el otoño, aparecen importantes áreas sembradas con trigo doble propósito (cosecha y cobertura) (Volante et al. 2007). Los bosques, que se caracterizan por ser semicaducifolios, presentan su mayor variabilidad interanual en el mes de junio, lo que puede estar asociado al efecto de las temperaturas sobre la actividad fotosintética y la caída de hojas. 
La descomposición de la señal de IVN en sus respectivos componentes leñosos y herbáceos permitió estimar el aporte de cada uno de ellos a la productividad total y describir su variabilidad temporal. Los resultados sugieren que a mayor cobertura arbórea se reduce la estacionalidad y se incrementa la productividad aérea total con respecto a las pasturas puras, aunque se reduce parcialmente la productividad aérea del pasto disponible para el ganado. De todas maneras, a pesar de que decrece la productividad subterránea, dado el balance negativo entre árboles y pastos, la productividad total entre ambos usos del suelo se asemeja. Sin embargo, dado que la metodología de Lu et al. (2003) supone que en los espacios cubiertos por el componente leñoso no hay presencia de herbáceas y viceversa, no se pudo estimar la productividad del pasto ubicado debajo de los árboles. Si bien Baldassini et al. (2018) mostraron que la productividad de Gatton Panic (Megathyrsus maximus var. Gatton) bajo los árboles de guayacán (Caesalpinea paraguariensis) (una de las especies arbóreas más frecuentes en estos sistemas) es aproximadamente la mitad que la observada a pleno sol, podría no observarse diferencias debajo de otros árboles que interceptan menos radiación. De esta manera, podríamos estar incurriendo en una subestimación de la productividad de pastos en aquellas situaciones en que la vegetación herbácea bajo las copas de los árboles sea abundante. De todas maneras, se han observado situaciones en donde la cobertura herbácea registrada bajo el componente leñoso era muy baja (Blanco et al. 2016; Baldassini et al. 2018), lo que podría estar asociado a una mala implantación de la pastura o a una remoción por parte del ganado al utilizar el sitio para descanso y como proveedor de sombra en condiciones de elevadas temperaturas (Cedeño et al. 2016; Tucker et al. 2008).

Los sistemas silvopastoriles surgieron como una alternativa promovida por ciertas provincias para la puesta en producción de áreas pertenecientesala Categoría II(Amarillo), en discordancia con lo postulado por la Ley N 26331 de Ordenamiento Territorial de los Bosques Nativos (OTBN). Sin embargo, a pesar de los efectos positivos ligados a la presencia del estrato arbóreo relacionados con la oferta de forraje en forma de follaje y/o frutos (e.g., guayacán[Caesalpiniaparaguariensis][Aronson and Toledo 1992]) y con la atenuación de las condiciones ambientales que operan sobre el bienestar animal (Chiossone et al. 2014), en la mayoría de los sistemas el componente forestal posee una importancia secundaria. A diferencia del Plan de Manejo de Bosques con Ganadería Integrada (MBGI, Borrás et al. 2017), que propone herramientas de manejo para compatibilizar la producción ganadera con la conservación del bosque, su biodiversidad y sus servicios ecosistémicos, la mayoría de los sistemas silvopastoriles actuales son básicamente ganaderos. El MBGI busca modificar la estructura del bosque mediante la reducción de los arbustos y la población de plantas leñosas a una densidad aceptable, preservando la diversidad de especies, la composición de plantas leñosas y las funciones del ecosistema (Rejžek et al. 2017). Así, para mantener al sistema dentro de los límites de la resiliencia resulta necesario considerar la reposición del estrato arbóreo y conservar las comunidades vegetales, manejando impactos de baja intensidad (severidad y frecuencia de rolados, agroquímicos y fuegos) y en escalas espaciales adecuadas a la conservación y reproducción (Carranza and Ledesma 2005; Casar 2016). Sin embargo, luego del rolado son muy pocos los árboles que quedan en pie, lo que dificulta el mantenimiento y regeneración futura de las especies leñosas. Esto se vio reflejado en la escasa cantidad de píxeles que presentaban una cobertura arbórea superior o igual al 25\%. Nuestros resultados sugieren que los sistemas con mayor proporción de árboles permitirían compatibilizar una producción ganadera intensiva con el mantenimiento de las funciones del ecosistema. Si bien estos sitios presentan una PPNA de pastos alrededor de $40 \%$ inferior que las pasturas puras, el aporte de los componentes leñosos determinó que no se observaran diferencias significativas en la productividad total.

La inclusión de información asociada a la eficiencia en el uso de la radiación y a relaciones PPNS/PPN, aporta no sólo información sustancial para concluir cuantitativamente en términos de la magnitud del cambio en unidades de materia seca, sino que también permite establecer un orden o ranking más apropiado de las coberturas y usos del suelo en cuanto a la productividad total. Esto resulta fundamental en situaciones, como las abordadas en este trabajo, en donde los usos del suelo presentan valores de EUR y relaciones PPNS/PPN muy dispares entre sí. De hecho, la EUR del maíz y del Gatton Panic, para ciertos momentos del año, fueron muy superiores a la utilizada para el bosque 
(alrededor de 1.9 g MS/MJ vs. 0.5 g MS/MJ), mientras que relación PPNS/PPN de los cultivos fue muy distinta a la utilizada para las pasturas (alrededor de 0.15 vs. 0.61). Si bien la radiación fotosintéticamente activa absorbida (RFAA) por el bosque a lo largo del año fue significativamente superior al resto de los usos del suelo, siendo entre 2 y 5 veces mayor que los cultivos agrícolas y pasturas, respectivamente (datos no mostrados), la consideración de ambas variables determinó que las diferencias en productividad entre usos del suelo sean mucho menores.

\section{CONCLUSIONES}

El uso del modelo de eficiencia en el uso de la radiación, junto con la aplicación de la metodología para la discriminación del IVN en sus respectivos componentes leñosos y herbáceos, resultaron en una mejora sustancial de las actuales estimaciones de la PPNA basadas en información espectral. La consideración de la PPNS permitió, a su vez, evaluar el impacto de los cambios en el uso del suelo sobre la PPN total. Sin embargo, ensayos experimentales en donde se monitoree la PPN resultan necesarios para evaluar estas estimaciones y reducir el nivel de incertidumbre. A su vez, futuras investigaciones podrían estar orientadas hacia ensayos decampo que permitan caracterizarlos cambios estacionales de la EUR de los distintos usos de suelo y sus controles climáticos, como así también de las relaciones PPNS/PPN. De esta manera, sería posible generar información más precisa de la dinámica de la PPN en función de las condiciones ambientales de un año particular.

AgradeCIMIENTOS. Este trabajo fue financiado por el CONICET, la UBA, FONCyT-PICT 2199 y por el Inter American Institute (IAI) for Global Change Research, que es financiado por la US National Science Foundation (Grant GEO-1128040), a través del proyecto CRN3095 (Bridging Ecosystem Services and Territorial Planning (BEST-P): A southern South American intiative). Agradecemos a Anta del Dorado, Sol González, Lucía Ciuffoli y Luciana Staiano por su contribuir en diferentes aspectos del trabajo.

\section{REFERENCIAS}

Aguiar, S., M. E. Mastrangelo, M. A. García Collazo, G. H. Camba Sans, C. E. Mosso, L. Ciuffoli, M. Schmidt, M. Vallejos, L. Langbehn, D. Cáceres, G. Merlinsky, J. M. Paruelo, L. Seghezzo, L. Staiano, M. Texeira, J. N. Volante, and S. R. Verón. 2018. ¿Cuál es la situación de la Ley de Bosques en la Región Chaqueña a diez años de su sanción? Revisar su pasado para discutir su futuro. Ecología Austral 28(02):400-417. https://doi.org/10.25260/EA.18.28.2.0.677.

Aronson, J., and C. S. Toledo. 1992. Caesalpinia paraguariensis (Fabaceae): Forage tree for all seasons. Econ Bot 46: 121-132. https://doi.org/10.1007/BF02930627.

Ávila, R., P. Barbera, L. Blanco, V. Burghi, J. P. De Battista, C. Frasinelli, K. Frigerio, L. Gándara, M. C. Goldfarb, et al. 2014. Gramíneas forrajeras para el subtrópico y el semiárido central de la Argentina, INTA, ISBN-978-987-521-551-1.

Baldassini, P., C. Despósito, G. Piñeiro, and J. M. Paruelo. 2018. Silvopastoral systems of the Chaco forests: Effects of trees on grass growth. Journal of Arid Environments 156:87-95. https://doi.org/10.1016/j.jaridenv.2018.05.008.

Baeza, S., J. Paruelo, and W. Ayala. 2011. Eficiencia en el uso de la radiación y productividad primaria en recursos forrajeros del este de Uruguay. Agrociencia Uruguay 15(2):48-59.

Bagnato, C., M. Texeira, G. García Accinelli, J. Volante, J. Paruelo. 2015. La fenoteca: una biblioteca espectral de firmas fenológicas y una propuesta metodológica para caracterizar el uso de la tierra. CISEN 4 IV Congreso Internacional de Servicios Ecosistémicos en los Neotrópicos, Mar del Plata, Agentina.

Baldi, G., S. R. Verón, and E. G. Jobbágy. 2013. The imprint of humans on landscape patterns and vegetation functioning in the dry subtropics. Global Change Biology 19(2):441-458. https:/ / doi.org/10.1111/gcb.12060.

Basualdo, M., N. Huykman, J. N. Volante, J. M. Paruelo, and G. Piñeiro. 2019. Lost forever? Ecosystem functional changes occurring after agricultural abandonment and forest recovery in the semiarid Chaco forests. Science of The Total Environment 650:1537-1546. https://doi.org/10.1016/j.scitotenv.2018.09.001.

Battaglia, M., and P. J. Sands. 1997. Modelling site productivity of Eucalyptus globulus in response to climatic and site factors. Aust J Plant Physiol 24:831-850. https://doi.org/10.1071/PP97065.

Baumann, M., I. Gasparri, M. Piquer'Rodríguez, G. Gavier Pizarro, P. Griffiths, P. Hostert, and T. Kuemmerle. 2017. Carbon emissions from agricultural expansion and intensification in the Chaco. Global Change Biology 23(5):19021916. https://doi.org/10.1111/gcb.13521.

Blackburn, W. J., and J. T. Proctor. 1983. Estimating photosynthetically active radiation from measured solar irradiance. Solar Energy 31(2):233-234. https://doi.org/10.1016/0038-092X(83)90087-7.

Blanco, L. J., J. M. Paruelo, M. Oesterheld, and F. N. Biurrun. 2016. Spatial and temporal patterns of herbaceous primary production in semi-arid shrublands: a remote sensing approach. J Veg Sci 27:716-727. https://doi.org/ 10.1111 /jvs.12398.

Cairns, M. A., S. Brown, E. H. Helmer, and G. A. Baumgardner. 1997. Root biomass allocation in the world's upland forests. Oecologia 111(1):1-11. https:/ / doi.org/10.1007/s004420050201. 
Calderini, D. F., M. F. Dreccer, and G. A. Slafer. 1997. Consequences of breeding on biomass, radiation interception and radiation-use efficiency in wheat. Field Crops Research 52(3):271-281. https://doi.org/10.1016/S0378-4290(96)03465$X$.

Cárcova, J., G. Abeledo, and M. López Pereira. 2004. Análisis de la generación del rendimiento: crecimiento, partición y componentes. Pp. 75-95 en E. H. Satorre, R. L. Vence, G. A. Slafer, E. B. de la Fuente, D. J. Miralles, M. E. Otegui and R. Savin (eds.). Producción de granos. Bases funcionales para su manejo. Editorial Facultad de agronomía. Universidad de Buenos Aires. Buenos Aires, Argentina.

Carranza, C., and M. Ledesma. 2005. Sistemas silvopastoriles en el Chaco Árido. IDIA XXI Forestales. INTA 8:240246.

Caruso, H., M. Camardelli, and S. Miranda. 2012. Efecto del método de desmonte sobre los indicadores de calidad del suelo y la condición de las pasturas en el Chaco Semiárido salteño. Agriscientia 29(2):99-105.

Casar, L. P. 2016. Silvopastoril, un sistema en expansión en la Argentina. RIA. Revista de Investigaciones Agropecuarias 42(2):120-124.

Ceballos, J. C., and L. O. Macedo. 2014. Uma base de dados de radiação solar na américa do sul, estimada por satélite (modelo GL1.2/CPTEC). V Congresso Brasileiro de Energia Solar, Recife, Brasil.

Chiossone, J. L., R. A. Vicini, A. Jacquet, and S. G. Ondo Misi. 2014. Comportamiento de Gatton Panic en Chaco (Argentina), mejoramiento en la utilización con suplementación y confinamiento en autoconsumo de silajes. XXII Congreso Internacional de Transferencia de Tecnología Agropecuaria, Consorcios de Ganaderos para Experimentación Agropecuaria. 3 y 4 de noviembre de 2014. Asunción, Paraguay.

Cleveland, R. B., W. S. Cleveland, and I. Terpenning. 1990. STL: A seasonal-trend decomposition procedure based on loess. Journal of Official Statistics 6(1):3.

Cornacchione, M., and J. P. Molina. 2008. Implantación de gramíneas subtropicales según fechas de siembra y acumulación y componentes de la materia seca. Revista Argentina de Producción Animal 28:349-543.

De León, M. 2004. Las pasturas subtropicales en la región semiárida central del país. Informe Técnico $\mathrm{N}^{\circ} 1$, Instituto Nacional de Tecnología Agropecuaria, Centro Regional Córdoba, ISSN 1668-2890.

Del Grosso, S., W. Parton, T. Stohlgren, D. Zheng, D. Bachelet, et al. 2008. Global potential net primary production predicted from vegetation class, precipitation, and temperature. Ecology 89(8):2117-2126. https://doi.org/10.1890/ 07-0850.1.

Facio, F. 2014. Caracterización de la relación entre índices de vegetación y la fracción de la radiación fotosintéticamente activa absorbida por canopeos con diferente estructura. Trabajo de Intensificación de la Licenciatura en Ciencias Ambientales - FAUBA. 14-06-2014.

Fehlenberg, V., M. Baumann, N. I. Gasparri, M. Piquer-Rodríguez, G. Gavier-Pizarro, and T. Kuemmerle. 2017. The role of soybean production as an underlying driver of deforestation in the South American Chaco. Global Environmental Change 45:24-34. https:/ / doi.org/10.1016/j.gloenvcha.2017.05.001.

Field, C. B., J. T. Randerson, and C. M. Malmstrom. 1995. Global net primary production: Combining ecology and remote sensing. Remote Sensing of Environment 51:74-88. https:/ / doi.org/10.1016/0034-4257(94)00066-V.

Fisher, B., R. K. Turner, and P. Morling. 2009. Defining and classifying ecosystem services for decision making. Ecological Economics 68(3):643-653. https://doi.org/10.1016/j.ecolecon.2008.09.014.

Galván, M. E. 2002. El cultivo de la soja en el NOA. Idia XXI (Buenos Aires-Argentina) 2(3):33-36.

García Collazo, M. A., A. Panizza, and J. M. Paruelo. 2013. Ordenamiento Territorial de Bosques Nativos: Resultados de la Zonificación realizada por provincias del Norte argentino. Ecología Austral 23(2):97-107.

Gasparri, N. I., and H. R. Grau. 2009. Deforestation and fragmentation of Chaco dry forest in NW Argentina (1972-2007). Forest ecology and Management 258(6):913-921. https://doi.org/10.1016/j.foreco.2009.02.024.

Gasparri, N. I., H. R. Grau, and J. G. Angonese. 2013. Linkages between soybean and neotropical deforestation: coupling and transient decoupling dynamics in a multi-decadal analysis. Global Environmental Change 23(6):1605-1614. https: //doi.org/10.1016/j.gloenvcha.2013.09.007.

Goetz, S. J., and S. D. Prince. 1996. Remote sensing of net primary production in boreal forest stands. Agricultural and Forest Meteorology 78(3-4):149-179. https://doi.org/10.1016/0168-1923(95)02268-6.

Gower, S. 1999. Direct and indirect estimation of leaf area index, $\mathrm{f}(\mathrm{APAR})$ and net primary production of terrestrial ecosystems. Remote Sensing of Environment 70:29-51. https://doi.org/10.1016/S0034-4257(99)00056-5.

Grau, H. R., N. I. Gasparri, and T. M. Aide. 2005. Agriculture expansion and deforestation in seasonally dry forests of north-west Argentina. Environ Conserv 32(2):140. https://doi.org/10.1017/S0376892905002092.

Grau, H. R., N. I. Gasparri, and T. M. Aide. 2008. Balancing food production and nature conservation in the Neotropical dry forests of northern Argentina. Glob Chang Biol 14(5):985-997. https://doi.org/10.1111/j.13652486.2008.01554.x.

Grigera, G., and M. Oesterheld. 2006. Forage production monitoring systems parameterization of fPAR and RUE. Global vegetation Workshop. The University of Montana, Missoula MT, USA, 7 al 10 de agosto de 2006.

Grigera, G., M. Oesterheld, and F. Pacín. 2007. Monitoring forage production for farmers' decision making. Agric Syst 94:637-648. https://doi.org/10.1016/j.agsy.2007.01.001.

Guerschman, J. P. 2005. Análisis regional del impacto de los cambios en el uso de la tierra sobre el funcionamiento de los ecosistemas en la región pampeana (Argentina). Presentada en la Facultad de Agronomía de la Universidad de Buenos Aires para la obtención del grado de Doctor en Ciencias Agropecuarias.

Haberl, H., K. H. Erb, F. Krausmann, V. Gaube, A. Bondeau, C. Plutzar, ..., and M. Fischer-Kowalski. 2007. Quantifying 
and mapping the human appropriation of net primary production in earth's terrestrial ecosystems. Proceedings of the National Academy of Sciences 104(31):12942-12947. https:/ / doi.org/10.1073/pnas.0704243104.

Hui, D., and R. B. Jackson. 2006. Geographical and interannual variability in biomass partitioning in grassland ecosystems: a synthesis of field data. New Phytologist 169:85-93. https:// doi.org/10.1111/j.1469-8137.2005.01569.x.

Huykman, N. 2011. Dinámica sucesional de áreas desmontadas a partir de sensores remotos en la provincia de Salta, Argentina. Licenciatura en Ciencias Ambientales, Facultad de Agronomía, Universidad de Buenos Aires. Pp. 55.

Jackson, R. B., J. Canadell, J. R. Ehleringer, H. A. Mooney, O. E. Sala, and E. D. Schulze. 1996. A global analysis of root distributions for terrestrial biomes. Oecologia 108(3):389-411. https://doi.org/10.1007/BF00333714.

Jobbágy, E. G., and R. B. Jackson. 2000. The vertical distribution of soil organic carbon and its relation to climate and vegetation. Ecological Applications 10(2):423-436. https://doi.org/10.1890/1051-0761(2000)010[0423: TVDOSO]2.0.CO;2.

Kunst, C., R. Ledesma, M. Castañares, M. Cornacchione, H. van Meer, and J. Godoy. 2014. Yield and growth features of Panicum maximum (Jacq.) var Trichoglume cv. Petrie (Green Panic) under woody cover, Chaco region, Argentina. Agrofor Syst 88:157-171. https://doi.org/10.1007/s10457-013-9663-4

Leadley, P. W., J. F. Reynolds, R. Flagler, and A. S. Heagle. 1990. Radiation utilization efficiency and the growth of soybeans exposed to ozone: a comparative analysis. Agricultural and Forest Meteorology 51(3-4):293-308. https: //doi.org/10.1016/0168-1923(90)90114-L.

Lu, H., M. Raupach, T. McVicar, and D. Barrett. 2003. Decomposition of vegetation cover into woody and herbaceous components using AVHRR NDVI time series. Remote Sensing of Environment 86:1-18. https://doi.org/10.1016/ S0034-4257(03)00054-3.

Martínez-Yrízar, A. 2009. Biomass distribution and primary productivity of tropical dry forests. Pp. 326-345 en S. H. Bullock, H. Mooney and E. Medina (eds.). Seasonally dry tropical forests. Cambridge University Press. https: //doi.org/10.1017/CBO9780511753398.013.

Matthews, E. 1983. Global vegetation and land use: new high-resolution data bases for climate studies. J Clim Appl Meteorol 22:474-500. https://doi.org/10.1175/1520-0450(1983)022\%3C0474:GVALUN\%3E2.0.CO;2.

McNaughton, S. J., M. Oesterheld, D. A. Frank, and K. J. Williams. 1989. Ecosystem-level patterns of primary productivity and herbivory in terrestrial habitats. Nature 341:6238:142-144. https://doi.org/10.1038/341142a0.

Miralles, D. J., G. A. Slafer, and V. Lynch. 1997. Rooting patterns in near-isogenic lines of spring wheat for dwarfism. Plant and Soil 197(1):79-86. https://doi.org/10.1023/A:1004207407979.

Mokany, K., R. Raison, and A. S. Prokushkin. 2006. Critical analysis of root: shoot ratios in terrestrial biomes. Global Change Biology 12(1):84-96. https://doi.org/10.1111/j.1365-2486.2005.001043.x.

Monteith, J. L. 1972. Solar radiation and productivity in tropical ecosystems. Journal of Applied Ecology 9:747-766. https://doi.org/10.2307/2401901.

Morello, J., S. D. Matteucci, A. F. Rodríguez, and M. F. Silva. 2012. Ecorregiones y complejos Ecosistémicos de Argentina. Orientación Gráfica Editora, Buenos Aires.

Moreno, A. 2012. Caracterización de cultivos del Noroeste Argentino a partir de Series Temporales del Índice de Vegetación. Ingeniería en Recursos Naturales y Medio Ambiente. Facultad de Ciencias Naturales, Universidad Nacional de Salta. Pp. 70.

Muchow, R. C., M. J. Robertson, and B. C. Pengelly. 1993. Radiation-use efficiency of soybean, mugbean and cowpea under different environmental conditions. Field Crops Research 32(1-2):1-16. https://doi.org/10.1016/03784290(93)90017-H.

Murray, F., G. Baldi, T. von Bernard, E. F. Viglizzo, and E. G. Jobbágy. 2016. Productive performance of alternative land covers along aridity gradients: Ecological, agronomic and economic perspectives. Agricultural Systems 149: 20-29. https://doi.org/10.1016/j.agsy.2016.08.004.

Oesterheld, M., J. Paruelo, and M. Oyarzabal. 2010. Estimación de la productividad primaria neta aérea a partir de diferencias de biomasa y de integración de la radiación absorbida. Bases ecológicas y tecnológicas para el manejo de pastizales. INIA-FPTA 26:113-120.

Olofsson, P., L. Eklundh, F. Lagergren, P. Jönsson, and A. Lindroth, A. 2007. Estimating net primary production for Scandinavian forests using data from Terra/MODIS. Advances in Space Research 39(1):125-130. https://doi.org/ 10.1016/j.asr.2006.02.031.

Otegui, M. E., M. G. Nicolini, R. A. Ruiz, and P. A. Dodds. 1995. Sowing date effects on grain yield components for different maize genotypes. Agronomy Journal 87(1):29-33. https://doi.org/10.2134/agronj1995.0002196200870001 0006x.

Oyarzabal, M., M. Oesterheld, and G. Grigera. 2011. ¿Cómo estimar la eficiencia en el uso de la radiación mediante sensores remotos y cosechas de biomasa? Un ejemplo local. Pp.121-135 en A. Altesor, W. Ayala and J. M. Paruelo (eds.). Bases ecológicas y tecnológicas para el manejo de pastizales. INIA, Serie FPTA No 26, Uruguay.

Paruelo, J. M., H. E. Epstein, W. K. Lauenroth, and I. C. Burke. 1997. ANPP estimates from NDVI for the Central Grassland Region of the United States. Ecology 78:953-958. https://doi.org/10.1890/0012-9658(1997)078[0953: AEFNFT]2.0.CO;2.

Paruelo, J. M., J. P. Guerschman, and S. R. Verón. 2005. Expansión agrícola y cambios en el uso del suelo. Ciencia Hoy 15(87):14-23.

Paruelo, J. M. 2011. Valoración de Servicios Ecosistémicos y planificación del uso del territorio. ¿Es necesario hablar de dinero? Pp. 121-140 en P. Laterra, E. G. Jobbágy and J. Paruelo (eds.). Valoración de Servicios Ecosistémicos. 
Conceptos, herramientas y aplicaciones para el ordenamiento territorial. INTA. Buenos Aires.

Paruelo, J. M., and M. Vallejos. 2013. Ecosystem Services related to C dynamics: its evaluation using Remote Sensing techniques. C. Di Bella, D. Alcaraz Segura and J. Straschnoy (eds.). Earth Observation of Ecosystem Services. Taylor and Francis Books.

Pérez, H. 2005. Características de las especies forrajeras adaptadas a las condiciones del Noroeste del país. Pp. 33-41 en Forrajes. Córdoba.

Pettorelli, N., J. O. Vik, A. Mysterud, J. M. Gaillard, C. J. Tucker, and N. C. Stenseth. 2005. Using the satellite-derived NDVI to assess ecological responses to environmental change. Trends in Ecology and Evolution 20(9):503-510. https: //doi.org/10.1016/j.tree.2005.05.011.

Piñeiro, G., M. Oesterheld, J. M. Paruelo. 2006. Seasonal variation in aboveground production and radiation use efficiency of temperate rangelands estimated through remote sensing. Ecosystems 9:357-373. https://doi.org/ 10.1007/s10021-005-0013-x.

Piquer-Rodríguez, M., S. Torella, G. Gavier-Pizarro, J. Volante, D. Somma, R. Ginzburg, and T. Kuemmerle. 2015. Effects of past and future land conversions on forest connectivity in the Argentine Chaco. Landscape Ecology 30(5): 817-833. https://doi.org/10.1007/s10980-014-0147-3.

Prause, J., G. Arce de Caram, and P. N. Angeloni. 2003. Variación mensual en el aporte de cuatro especies forestales nativas del Parque Chaqueño Humado (Argentina). Revista de Ciencias Forestales - Quebracho 10:39-45.

Primer Inventario Nacional de Bosques Nativos de Argentina. 2005. Proyecto Bosques Nativos y Áreas Protegidas. Secretaría de Ambiente y Desarrollo Sustentable, Buenos Aires.

Riffel, S. L., and J. C. Elizalde. 2008. Invernada en regiones no tradicionales. Bs. As. Revista Braford 24(59):36-41.

Roca Cedeño, A. J., P. J. Lascano Armas, C. N. Arcos Álvarez, E. Z. Sánchez Santana, R. V. Guevara Viera, J. C. Vera Cedeño, V. G. Serpa García, C. U. Iñiguez Gutiérrez, G. E. Guevara Viera, and L. M. Curbelo Rodríguez. 2016. Influencia del algarrobo en la conducta y producción de leche de vacas en pastoreo. I. Período de Seca. Revista de Producción Animal 28(1):1-9.

Rueda, C. V., G. Baldi, S. R. Verón, and E. G. Jobbágy. 2013. Apropiación humana de la producción primaria en el Chaco Seco. Ecología Austral 23(1):44-54.

Ruimy, A., B. Saugier, and G. Dedieu. 1994. Methodology for the estimation of terrestrial net primary production from remotely sensed data. Journal of Geophysical Research 99:5263-5283. https://doi.org/10.1029/93JD03221.

Sala, O. E., and A. T. Austin. 2000. Methods of estimating aboveground net primary productivity. Pp. 31-43 in Methods in Ecosystem Science. Springer, New York, NY. https:/ / doi.org/10.1007/978-1-4612-1224-9_3.

SAyDS. 2005. Primer Inventario Nacional de Bosques Nativos. Proyecto Bosques Nativos y Áreas Protegidas BIRF 4085-AR 1998-2001. Informe Regional Parque Chaqueño.

Seaquist, J. W., L. Olsson, and J. Ardö. 2003. A remote sensing-based primary production model for grassland biomes. Ecological Modelling 169(1):131-155. https:// doi.org/10.1016/S0304-3800(03)00267-9.

Sellers, P. J., J. A. Berry, G. J. Collatz, C. B. Field, and F. G. Hall. 1992. Canopy reflectance, photosynthesis, and transpiration. III. A reanalysis using improved leaf models and a new canopy integration scheme. Remote Sensing of Environment 42:187-216. https:// doi.org/10.1016/0034-4257(92)90102-P.

Staiano, L. 2016. Una caracterización de los cambios funcionales de la vegetación asociados a la transformación del territorio en el noroeste argentino. Licenciatura en Ciencias Ambientales, Facultad de Agronomía, Universidad de Buenos Aires. Pp. 89.

Ting, K. C., and G. A. Giacomelli. 1987. Availability of solar photosynthetically active radiation. Transactions of the ASAE 30(5):1453-1457. https:/ / doi.org/10.13031/2013.30585.

Tucker, C. B., A. R. Rogers, and K. E. Schütz. 2008. Effect of solar radiation on dairy cattle behaviour, use of shade and body temperature in a pasture-based system. Applied Animal Behaviour Science, 109(2-4):141-154. https://doi.org/ 10.1016/j.applanim.2007.03.015.

Vallejos, M., J. N. Volante, M. J. Mosciaro, L. M. Vale, M. L. Bustamante, and J. M. Paruelo. 2015. Transformation dynamics of the natural cover in the Dry Chaco ecoregion: a plot level geo-database from 1976 to 2012 . Journal of Arid Environments 123:3-11. https://doi.org/10.1016/j.jaridenv.2014.11.009.

Vargas Gil, J. R., and A. Vorano. 1988. Suelos y vegetación. Desmonte y habilitación de tierras en la Región Chaqueña Semiárida. Red de Cooperación Técnica en Uso de los Recursos Naturales de la Región Chaqueña Semiárida de Argentina-Bolivia-Paraguay. FAO, Oficina Regional para América Latina y el Caribe. Santiago, Chile. Pp. 22-31.

Verón, S., E. Jobbágy, I. Gasparri, P. Kandus, M. Easdale, et al. 2011. Complejidad de los servicios ecosistémicos y estrategias para abordarla. P. Laterra, E. Jobbágy and J. Paruelo (eds.). Valoración de Servicios Ecosistémicos. Conceptos, herramientas y aplicaciones para el ordenamiento territorial. Ediciones INTA.

Veroustraete, F., J. Patyn, and R. B. Myneni. 1996. Estimating net ecosystem exchange of carbon using the Normalised Difference Vegetation Index and an ecosystem model. Remote Sensing of Environment 58:115-130. https://doi.org/ 10.1016/0034-4257(95)00258-8.

Viglizzo, E. F., F. C. Frank, L. V. Carreño, E. G. Jobbágy, H. Pereyra, et al. 2011. Ecological and environmental footprint of 50 years of agricultural expansion in Argentina. Global Change Biology 17(2):959-973. https://doi.org/10.1111/ j.1365-2486.2010.02293.x.

Volante, J. N., A. R. Bianchi, and H. P. Paoli. 2001. Monitoreo de cultivos extensivos del NOA a partir de sensores remotos. Campaña agrícola de verano 2000-2001. INTA EEA Salta. Pp. 25. URL: www.inta.gov.ar/region/noa/prorenoa/.

Volante, J. N., A. Bianchi, H. Paoli, and Y. Noé. 2006. Análisis de la Dinámica del Uso del Suelo Agrícola del Noroeste 
Argentino Mediante Teledetección y Sistemas de Información Geográfica Período 2000-2005. Ediciones INTA 2009. Volante, J. N., D. Alcaraz-Segura, M. J. Mosciaro, E. F. Viglizzo, and J. M. Paruelo. 2012. Ecosystem functional changes associated with land clearing in NW Argentina. Agriculture, Ecosystems and Environment 154:12-22. https://doi.org/ 10.1016/j.agee.2011.08.012.

Volante, J. N., M. J. Mosciaro, G. I. Gavier-Pizarro, and J. M. Paruelo. 2016. Agricultural expansion in the Semiarid Chaco: Poorly selective contagious advance. Land Use Policy 55:154-165. https://doi.org/10.1016/j.landusepol.20 16.03.025. 